\title{
Haftung aufgrund unterlassener pränataler Diagnostik
}

\author{
Das Obergericht des Kantons Bern hatte zu entscheiden, ob einem wegen unter- \\ lassener pränataler Diagnostik nicht abgetriebenen, sondern behindert zur Welt ge- \\ kommenen Kind eine Genugtuung zu bezahlen ist (Wrongful-life-Klage) [1]*.
}

Ursina Pally Hofmann

Stv. Leiterin Rechtsdienst FMH

* Die Literaturangaben finden sich im Internet unter www.saez.ch $\rightarrow$ Aktuelle Nummer oder $\rightarrow$ Archiv $\rightarrow 2013 \rightarrow 15$.

Korrespondenz:

Dr. iur. Ursina Pally Hofmann Rechtsanwältin

Rechtsdienst FMH

Elfenstrasse 18

CH-3000 Bern 15

Tel. 0313591111

Fax 0313591112

ursina.pally[at]fmh.ch

\section{Sachverhalt und Prozessgeschichte}

Die schwangere Frau begab sich nach der 12. Schwangerschaftswoche zur Frauenärztin. Dieser war bekannt, dass für die ungeborene Tochter ein erhöhtes Risiko bestand, an einer vererblichen Stoffwechselerkrankung zu leiden. Deshalb hätte sie bei der schwangeren Frau weitergehende pränatale Untersuchungen vornehmen müssen, was sie unterliess. Die Vorinstanz [2] * hatte festgestellt, dass die Mutter mit grösster Wahrscheinlichkeit abgetrieben hätte, wenn sie von der Erkrankung ihrer Tochter gewusst hätte. Der eingeklagte Anspruch der Mutter auf Genugtuung wurde von der Vorinstanz bejaht. Derjenige des Kindes mit der Begründung, dass ein Vergleich des behinderten Lebens mit einem hypothetisch gesunden Leben nicht zulässig sei, verneint. Die Klägerinnen (Mutter und Tochter) gelangten in der Folge ans Obergericht des Kantons Bern. Nachfolgend wird dargelegt, wie es bei Vorliegen desselben Sachverhaltes zu dieser auf den ersten Blick erstaunlichen Unterscheidung zwischen den Ansprüchen von Mutter und Kind kommt.

\section{Haftungsvoraussetzungen und Genugtuung für die Mutter}

Fälle, in denen es wegen einer fehlerhaften Durchführung familienplanerischer Massnahmen (wrongful pregnancy, wrongful conception) zu einer ungeplanten Schwangerschaft kommt, eine Schwangerschaft wegen fehlerhafter genetischer Beratung nicht verhindert oder eine Schwangerschaft wegen fehlerhafter pränataler Diagnostik nicht abgebrochen wird (wrongful birth), führen regelmässig zu Schadenersatzund/oder Genugtuungszahlungen, wenn die Haftungsvoraussetzungen erfüllt sind und die Mutter - in manchen Fällen auch der Vater - in eigenem Namen klagen. Kontrovers diskutiert und schwieriger zu beantworten ist die Frage, ob auch ein ursprünglich behindertes Kind, das wegen einer unterlassenen oder der fehlerhaft durchgeführten Pränataldiagnose nicht abgetrieben wurde, Anspruch auf Schadenersatz und Genugtuung hat (wrongful life).

Ein Arzt kann im Zusammenhang mit der Geburt eines ungewollten Kindes grundsätzlich haftbar gemacht werden, wenn die folgenden vier Voraussetzungen erfüllt sind [3]:

\section{Schaden oder immaterielle Unbill}

Als Schaden im Zusammenhang mit der Geburt eines ungewollten Kindes wird nicht die Existenz des Kindes angesehen, sondern die Kosten, die mit seiner Erziehung und Pflege verbunden sind. Ein Schaden berechnet sich nach der Differenztheorie. Der Körperschaden muss zu einer unfreiwilligen Vermögenseinbusse führen. Es wird also verglichen zwischen dem Vermögensstand ohne und Vermögensstand mit schädigendem Ereignis.

Die durch die Geburt eines behinderten Kindes das zwar grundsätzlich gewollt war, aber nur wenn es gesund gewesen wäre - verursachte immaterielle (seelische bzw. moralische) Unbill der Mutter führt zu einer Genugtuungszahlung. Dies deshalb, weil ihr Selbstbestimmungsrecht verletzt wurde, da sie die Schwangerschaft nicht abbrechen konnte. Zudem ist sie durch die Pflege des behinderten Kindes sowohl in ihrer eigenen Lebensführung als auch in der Beziehung zum Kind beeinträchtigt.

\section{Vertragsverletzung}

Die schwangere Frau schliesst mit der Ärztin einen Behandlungsvertrag ab, der eine sorgfältige Behandlung vorsieht. Zu dieser gehört auch die Vornahme der indizierten pränatalen Diagnostik, sofern die schwangere Frau nach genügender Aufklärung nicht selbst darauf verzichtet. Wird die indizierte Diagnostik nicht vorgenommen, behandelt die Ärztin unsorgfältig und verletzt den Vertrag.

\section{Kausalzusammenhang}

Ein Kausalzusammenhang zwischen dem Unterlassen der pränatalen Diagnostik und der Geburt des behinderten Kindes liegt vor, wenn die Geburt durch die sorgfältige Behandlung hätte verhindert werden können. Davon kann bei einer Schwangerschaft mit einem behinderten Kind nur ausgegangen werden, wenn ein Schwangerschaftsabbruch legal möglich war, und wenn die schwangere Frau die Schwangerschaft bei Kenntnis der Behinderung abgebrochen hätte [4]. Sind diese beiden Bedingungen nicht erfüllt, käme es auch dann zur Geburt eines behinderten Kindes, wenn der Arzt die Diagnostik sorgfältig durchgeführt hätte. Im beurteilten Fall wurde davon ausgegangen, dass die medizinische Indikation von Art. 119 
Abs. 1 StGB vorlag, weil die Mutter durch Schwangerschaft und Geburt des behinderten Kindes in eine schwere seelische Notlage geraten war. Offenbar gelang der Mutter der Nachweis, dass sie in Kenntnis der Behinderung abgetrieben hätte. Dieser Nachweis dürfte immer dann gelingen, wenn nicht aufgrund eines bisherigen Verhaltens der betreffenden Frau - in einer vergleichbaren Situation schon einmal nicht abgetrieben zu haben - oder ihrer religiösen Ausrichtung auf das Gegenteil geschlossen werden kann.

\section{Verschulden}

Im vertraglichen Verhältnis wird das Vorhandensein des Verschuldens vermutet. Es gelang der Ärztin vorliegend nicht, diese Vermutung zu widerlegen.

Da alle vier Haftungsvoraussetzungen erfüllt waren, wurde der Genugtuungsanspruch der Mutter bejaht.

\section{Anspruch des Kindes}

Die Diskussion um den Anspruch des behinderten Kindes fokussiert sich auf die Definition des Schadens [5] (Vergleich von behindertem Leben und Nichtexistenz) und die Vertragsverletzung oder die Verletzung eines Rechtsgutes (körperliche Unversehrtheit oder Recht auf Nichtexistenz).

\section{Lehre und Rechtsprechung}

In der juristischen Literatur wird die Diskussion über den Anspruch des Kindes kontrovers geführt. Einige Juristen [6] gehen davon aus, dass sich die Pflicht der Ärztin, die pränatale Diagnostik sorgfältig durchzuführen, auch auf das Ungeborene erstreckt, weil dessen zukünftige Rechtsgüter direkt betroffen sind. Der nach schweizerischem Recht legale Schwangerschaftsabbruch mache klar, dass die Geburt eines behinderten Kindes von der Mutter als Nachteil gesehen werden könne. Dieser Ansicht könne auch das behinderte Kind selbst sein. Aus Gründen der Billigkeit und Gleichheit müsse dem Kind ein Anspruch auf Entschädigung seines Schadens zustehen [7]. Es wird darauf hingewiesen, dass die Mutter zwar eine Interessenabwägung machen dürfe (Relativierbarkeit des Lebens des Kindes), das Kind hingegen kein Recht darauf haben soll, nicht geboren zu werden, selbst wenn zu erwarten sei, dass seine Behinderung zu einem unerträglichen Leben führen wird (Absolutheit des Lebens des Kindes) [8]. Anzumerken ist hier, dass die medizinische Indikation für einen Abbruch der Schwangerschaft gegeben ist, wenn der Mutter die Gefahr einer schwerwiegenden körperlichen Schädigung droht oder sie sich in einer schweren seelischen Notlage befindet. Wenn man dem Kind das Recht darauf, abgetrieben zu werden, zugestehen würde, müsste man entscheiden, ob sein behindertes Leben unerträglich sein wird. Es ist offensichtlich, dass diese Einschätzung für einen Richter oder die Mutter nur äusserst schwierig, die Frage nach der Gefahr einer Schädigung der Mutter im Vergleich dazu wesentlich einfacher zu beantworten ist. Eine Genugtuungszahlung für das Kind kann nur dann geleistet werden, wenn man das Recht des behinderten Kindes, nicht geboren zu werden, bejaht [9]. Die Existenz eines solchen Rechts wird von verschiedenen Autoren verneint [10].

Die Rechtsprechung in den umliegenden europäischen Staaten ist nicht einheitlich. Einzig in Frankreich [11] wurde der Anspruch des Kindes mehrmals bejaht. Später wurde in Frankreich ein Gesetz erlassen, nach dem niemand ausschliesslich aufgrund seiner Geburt Schadenersatz geltend machen kann [12]. Eine entsprechende gesetzliche Regelung existiert auch in England [13]. Vor dem Erlass dieses Gesetzes wurde ein vor den englischen Gerichten eingeklagter Anspruch abgewiesen, weil eine Schadenberechnung nach der Differenztheorie nicht möglich sei [14]. Das geschah auch in Österreich [15] mit derselben Begründung. Ein Anspruch des behinderten Kindes wurde vom deutschen Bundesgerichtshof ebenfalls abgelehnt [16]. Das Kind könne nicht vorbringen, es wäre besser nicht geboren worden.

\section{Begründung des Obergerichts Bern}

Das Obergericht [17] des Kantons Bern führte aus, dass eine Voraussetzung für die immaterielle Unbill und damit für die Genugtuungszahlung die Verletzung des Rechts auf körperliche Unversehrtheit sei. Die körperliche Unversehrtheit des Kindes sei im vorliegenden Fall nicht durch die Ärztin beeinträchtigt worden, und es habe keine Möglichkeit bestanden, auf die genetisch bedingte Erkrankung Einfluss zu nehmen. Deshalb liege keine Körperschädigung vor. Man könne nicht ausser Acht lassen, dass die einzige Alternative die Nichtexistenz des Kindes gewesen wäre. Der Begriff der Schädigung verlange einen Vergleich mit einem günstigeren Alternativzustand. Da ein gesundes Leben in keinem Falle möglich gewesen wäre, gäbe es keine Alternative ausser der Nichtexistenz. Das Gericht ging davon aus, dass eine Trennung von Behinderung und Leben des Kindes nicht möglich ist, weshalb es das Vorliegen eines durch die Ärztin verursachten Körperschadens und damit einer materiellen Unbill verneinte.

Das Obergericht prüfte dann, ob sich aus der Verletzung des Persönlichkeitsrechts auf Nichtexistenz eine Genugtuungszahlung ableiten lasse. Es kam zum Schluss, dass in der Schweiz zwar in bestimmten Fällen ein Anspruch der Mutter auf Abtreibung bestehe, ein Anspruch des Kindes auf «nicht geboren zu werden» dem schweizerischen Recht aber nicht bekannt sei. Da das Ungeborene im Gegensatz zu seiner Mutter kein diesbezügliches Wahlrecht habe, lasse sich ein Recht auf Nichtexistenz des Kindes nicht aus dem Recht der Mutter ableiten. Auch habe die Ärztin gegenüber dem Kind keine vertragliche Pflicht, es nicht zur Welt kommen zu lassen.

Der Anspruch des Kindes auf Genugtuung wurde vom Obergericht verneint. Das Urteil wurde nicht ans Bundesgericht weitergezogen und ist rechtskräftig. Das Bundesgericht hatte noch keinen solchen Fall zu beurteilen. 


\section{Literatur}

1 Urteil der 2. Zivilkammer des Obergerichts des Kantons Bern vom 2. Mai 2011.

2 Urteil des Gerichtspräsidenten 1 des Gerichtskreises VIII Bern-Laupen vom 18. Oktober 2010.

3 Art. 41 OR i.V.m. Art. 97 i.V.m. Art. 298 OR.

4 Pally U. Arzthaftung mit den Schwerpunkten Schwangerschaftsbetreuung und Geburtshilfe, Diss. Zürich 2007, 53 f. m.w.H.

5 Pally, 25 ff.; Henri Torrione, Le Préjudice d'être né, HAVE 2006, $388 \mathrm{ff}$.

6 Thomas M. Mannsdorfer, Pränatale Schädigung, Diss. Fribourg 2000, Rz. 931 ff.; Christoph Müller, Arzthaftung für das unerwünschte behinderte Kind, AJP. 5/2003, 522 ff., $530 \mathrm{ff}$.

7 Chappuis G. L'indemnisation du préjudice découlant de la naissance d'un enfant non désiré: une simple question d'arithmétique? Quelques considérations à la lumière des solutions apportées par le droit français, HAVE 2006, 378 ff. ,381 und FN 22.

8 Picker E. Schadenersatz für das unerwünschte eigene Leben, «Wrongful life», Tübingen 1995, 40 ff.

9 Junker C. Pflichtverletzung, Kindesexistenz und Schadenersatz, Berlin 2002, Diss. Heidelberg 2000, 643,645 f., 654, $660 \mathrm{ff}$.
10 Melchert T. Ärztliche Haftpflicht bei Fällen von Wrongful life. Jusletter. 12.1.2004, Rz 2.

11 Urteil der Cour de cassation vom 17. November 2000, JCP G 2000, II, 10438, 2293-302.

12 Mannsdorfer T. Franz. Kassationshof, 28.11.2001, Zulässigkeit von Schadenersatz- und Genugtuungsansprüchen gegen sorgfaltswidrig handelnde Medizinalpersonen aufgrund der eigenen, von den Eltern aber «unerwünschen» Geburt, AJP 11/2002, 1360-6, 1365.

13 Rüetschi D. «Wrongful Life» - die französische Sichtweise, FamPra.ch 2/2001, p. 266 ff., 271.

14 McKay v. Essex AHA, (1982) QB 1166, 1192.

15 OHG 1OB91/99k vom 25. Mai 1999.

16 BGH, NJW 2002, 2636; BGH, VersR 1981, 481, die Alternative, das Leben des Kindes nicht entstehen zu lassen, haben nur die Eltern, nicht aber das Kind. Diese Beurteilung hat der BGH im Zusammenhang mit der Geltendmachung von Schadenersatz eines gesunden ungewollten Kindes vorgenommen.

17 Urteil der 2. Zivilkammer des Obergerichts des Kantons Bern vom 2. Mai 2011, Erwägung C. ii. 